\title{
Continuous glucose monitoring and insulin pump therapy in pregnant women with type 1 diabetes mellitus
}

\author{
Izabela Lason ${ }^{\circledR}$, Katarzyna Cyganek® ${ }^{\circledR}$, Przemyslaw Witek, Bartlomiej Matejko, \\ Maciej T. Malecki, Jan Skupien \\ Department of Metabolic Diseases, University Hospital, Cracow, Poland \\ Department of Metabolic Diseases, Jagiellonian University Medical College, Cracow, Poland
}

\begin{abstract}
Objectives: We examined the impact of continuous subcutaneous insulin infusion (CSII) and continuous glucose monitoring systems (CGM) during pregnancy in women with pre-gestational type 1 diabetes (T1DM) on glycemic control and subsequent adverse outcomes.

Material and methods: In this observational, one-center study we analyzed records of consecutive 109T1DM pregnancies (2016-2017). The final analyzed group consisted of 81 singleton pregnancies who met inclusion and exclusion criteria. We searched for the association between the use of CSII with or without CGM and pregnancy planning with glycated hemoglobin A1c (HbA1c) through pregnancy and after delivery as well as maternal and infant outcomes.

Results: Patients using CSII and CGM vs CSII without CGM and MDI (multiple daily injections) users had the lowest HbA1C levels during and after pregnancy $\left(5.3 \%, 5.3 \%, 5.2 \%\right.$ and $5,5 \%$ in the $1^{\text {st }}, 2^{\text {nd }}, 3^{\text {rd }}$ trimester and postpartum visit, $p=0.003$, $p=0.030, p=0.039$ and $p=0.002$, respectively). Patients treated with insulin pumps with CGM and additional functions of automatic insulin delivery suspension on low glucose level (SLG) or predictive low glucose suspend (PLGS) during the third trimester and after pregnancy achieved a significantly lower HbA1c than the other CSII patients. We did not find any differences between the study groups in gestational age at delivery, preterm births, birth weight or macrosomia risk. Despite very good glycemic control, the risk of macrosomia remained high (19.7\%).

Conclusions: The use of pumps equipped with CGM, especially with automatic insulin delivery suspension, may improve glycemic control in pregnant T1DM women. The proportion of macrosomia remained high.

Key words: type 1 diabetes; pregnancy; continuous glucose monitoring; insulin pump; macrosomia
\end{abstract}

Ginekologia Polska 2021; 92, 10: 675-681

\section{INTRODUCTION}

Maintaining target glucose and glycated hemoglobin $\mathrm{A}_{1 \mathrm{c}}\left(\mathrm{HbA}_{1 \mathrm{c}}\right)$ levels in normoglycemic states while pregnant with diabetes type 1 is essential to reduce the risk of fetal hyperglycemia exposure. During this same period, it is also important to limit maternal hypoglycemic episodes. Both objectives are considered critical to reducing the risk of poor outcomes in both mother and neonate [1, 2].

However, the burden of macrosomia in pregnancies in women with pre-gestational type 1 diabetes (T1DM) remains still higher compared to the general population, despite in-target or near-target glycemic control and the risk of macrosomia remained glucose-dependent [3].
The high risk of macrosomia for in-target $(<6.0 \%)$ hemoglobin $\mathrm{A}_{1 \mathrm{c}}\left(\mathrm{HbA}_{1 \mathrm{c}}\right)$ may be driven by hyperglycemic spikes missed in routine self-monitoring of blood glucose (SMBG) performed with a glucose meter.

Inadequate blood glucose management during pregnancy was linked not only to macrosomia but also to other adverse pregnancy outcomes in mothers and infants. These risks were extended to potential obesity, diabetes and cardiovascular disease later in child's life [4-6]. Approximately half of the pregnant women with diabetes in the United Kingdom do not reach their HbA1c targets [7]. The improvement of pre-pregnancy care and the promotion of pregnancy planning may be adequate and effective. However, 
a new challenge emerges for the women reaching the recommended glycemic target.

Continuous glucose monitoring systems (CGM) are becoming more widespread in recent years. They offer a complete, 24-hour glucose profile capturing all blood glucose fluctuations. The measurement takes place in the subcutaneous tissue with a reduced need for fingerstick calibration, where rapid changes in glucose concentration mirror the changes in the blood. Modern personal insulin pumps augmented with CGM sensors with functions like automatic insulin delivery suspension on low glucose level (SLG) or predictive low glucose suspend (PLGS) offer additional benefits for their users. In a cost-utility analysis performed in Sweden, it was concluded that the use of a SLG pump was effective in T1DM patients with poorly controlled diabetes and in those with a high risk of hypoglycemia [8].

A recent CONCEPTT study found that the prolonged use of CGM through pregnancy in women with pregestational T1DM resulted in the reduction in $\mathrm{HbA} 1 \mathrm{c}$, more time spent within target glucose levels, less time spent above the target and reduced glycemic variability [9]. Such improvements translated into better neonatal outcomes, particularly in the lower incidence of macrosomia and neonatal hypoglycemia.

The use of CGM and sensor-augmented insulin pumps are becoming a standard of care in pregnant women with T1DM [10, 11]. Here, we report our initial experience with the use of CGM in pregnant T1DM women in the Lesser Poland region.

\section{Objectives}

Our aim was to examine the impact of the use of CGM and continuous subcutaneous insulin infusion (CSII) on glycemic control and the risk of maternal and infant adverse outcomes as compared to MDI during pregnancy in women with pre-gestational T1DM.

\section{MATERIAL AND METHODS}

This study was performed at the Clinical Department of Metabolic Diseases, University Hospital in Krakow, Poland, a tertiary regional referral center for diabetes. In this single-center retrospective clinical observation, we included all consecutive women with pre-existing T1DM who were registered between the years 2016 and 2017, and their data was collected at the time of the first antenatal visit. All participants were Caucasians and residents of southeastern Poland. Data from their medical records including pregestational and clinical data were collected during the first pregnancy visit, as described earlier [3, 12]. We included all consecutive women diagnosed with T1DM at least one year before conception. The study participants were followed during their pregnancy and up to 3-6 months postpartum through the end of 2018. T1DM was diagnosed based on the World Health Organization criteria and acute onset with typical symptoms, ketosis or ketoacidosis at the time of diagnosis and insulin dependence from the onset. Autoantibodies were not routinely tested and were not required for a T1DM diagnosis.

Pregnant or pregnancy planning women received an intensive diabetes management program, which involved education on diet, physical activity, glycemic goals, SMBG (self-monitoring of blood glucose), frequent outpatient visits, and if necessary, hospital admission. The educational program was adjusted for the appropriate insulin treatment regimen: multiple daily injections (MDI) or CSII with a personal insulin pump. Whenever CGM was available, its use was included in the education program. A woman with T1DM referred to the center before conception was considered pregnancy planning. If the first visit was registered after conception, such a pregnancy was considered not planned.

According to the guidelines of the Polish Diabetes Association, the target $\mathrm{HbA} 1 \mathrm{c}$ was $<6.0 \%$, self-monitored glucose $3.9-5.0 \mathrm{mmol} / \mathrm{L}(70-90 \mathrm{mg} / \mathrm{dL})$ at fasting states, $<6.7 \mathrm{mmol} / \mathrm{L}$ $(<120 \mathrm{mg} / \mathrm{dL}) 60$ minutes after meals and $>3.3 \mathrm{mmol} / \mathrm{L}$ (> $60 \mathrm{mg} / \mathrm{dL}$ ) between 2 and 4 am [13]. In 2017, the targets were changed to $\mathrm{HbA}_{1 \mathrm{c}}<6.5 \%$ in $1^{\text {st }}$ trimester and $<6.0 \%$ in $2^{\text {nd }}$ and $3^{\text {rd }}$ trimester, self-monitored glucose $3.9-5.0 \mathrm{mmol} / \mathrm{L}$ $(70-90 \mathrm{mg} / \mathrm{dL})$ at fasting states, $<7.8 \mathrm{mmol} / \mathrm{L}(<140 \mathrm{mg} / \mathrm{dL})$ 60 minutes after meals and $3.9-5.0 \mathrm{mmol} / \mathrm{L}(70-90 \mathrm{mg} / \mathrm{dL})$ between 2 and 4 am [14]. All women had diabetes and control obstetric visits every four weeks throughout the pregnancy. The recommended standard calorie intake was about $30-35 \mathrm{kcal} / \mathrm{kg}$ of body weight and depended on the body weight, height, physical activity and age [13]. The patients were advised to consume $40-50 \%$ of calories from carbohydrates, $20-30 \%$ from fats and $30 \%$ from proteins. The recommended weight gain depended on baseline body weight (11.3-15.9 kg for women with normal weight, 6.8-11.4 kg for overweight women, 4.5-9.1 kg for women with obesity and up to $18 \mathrm{~kg}$ for patients with $\mathrm{BMI}<19.8 \mathrm{~kg} / \mathrm{m}^{2}$ ) [13-15]. Excess weight gain was managed by reducing food intake. Ketone levels were monitored daily from a morning urine sample.

In the Clinical Department of Metabolic Diseases, there were insulin pumps available for rent to T1DM pregnant women or those who were planning a pregnancy. The insulin pumps and sensors came from a donation program sponsored by "The Great Orchestra of Christmas Charity" Foundation (The Orchestra Foundation), one of the largest charities in Poland. The costs of the insulin pump and CGM are not reimbursed for patients under the age of 26 in Poland. The specialists in our out-patient clinic recommended and prescribed CSII or sensor-augmented insulin pump (SAP) therapy due to pregnancy or in women planning to become pregnant. Women in our study received personal 
insulin pumps and CGM (transmitter and sensors) free of charge. There were two pump models: automatic insulin delivery suspension on low glucose level (SLG) - Paradigm Veo or predictive low glucose suspend (PLGS) - MiniMed640G. Patients were strongly encouraged to use the CSIl regimen as well as CGM during pregnancy, although this was limited due to patient compliance. The choice of insulin pump model, SLG or PLGS, was usually based on the current availability. The use of MDI was based on the patient's personal preferences.

We excluded from the current analysis women who experienced a miscarriage (before $22^{\text {nd }}$ week of gestation, $\mathrm{n}=6$ ) or with incomplete follow-up data, specifically without a documented postnatal visit with a recorded gestational age and birth weight. Only singleton pregnancies were analyzed. The baseline characteristics were recorded at the first visit. They included age, T1DM duration, preconception body weight, body mass index (BMI), and the presence of diabetes complications such as retinopathy diagnosed by an ophthalmologist, decreased glomerular filtration or albuminuria. Every woman had their $\mathrm{HbA}_{1 \mathrm{c}}$ level tested at the first, second and third trimester, as well as 3-6 months after delivery. In addition, the preconception $\mathrm{HbA}_{1 \mathrm{c}}$ was also obtained either from the patient's past medical records or it was measured during their pregnancy planning visit.

The following endpoints were considered in the analysis: $\mathrm{HbA}_{1 \mathrm{c}}$ level and its change throughout the pregnancy and after delivery, the maternal weight gain during pregnancy, the birth weight and the presence of macrosomia (> $4000 \mathrm{~g}$ ) in term pregnancies, the low birth weight $(<2500 \mathrm{~g})$, the frequency of preterm deliveries ( $<37^{\text {th }}$ week of gestation), the frequency of caesarean deliveries, infant deaths and congenital malformations. We searched for the association between these variables and the specific model of insulin therapy (the use of insulin pump with or without CGM or MDI regimen) as well as pregnancy planning. We were unable to assess the newer parameters of glucose control quality like time in range (TIR), time below range (TBR) and above range (TAB), glycaemic variability assessed by coefficient variation (\%CV) and GMI (glucose management indicator) because only some of the patients used CGM [16].

The data is reported as counts and percentages for categorical variables or medians with quartiles for continuous variables (more appropriate for small samples). Proportions were compared using the chi-squared test or Fisher exact test, when applicable. Differences between medians were compared using the Wilcoxon rank sum test. Shapiro-Wilk test was used to assess normality of the distribution. Bartlett's test was used to assess homogeneity of variances. To compare the mean of more than two groups we used ANOVA test or Kruskal-Wallis rank sum test as nonparametric equivalent. The differences between mean levels of $\mathrm{HbA}_{1 \mathrm{c}}$ were tested in a multiple repeated measures regression model that implemented an unstructured covariance matrix. Data analysis was performed in SAS 9.4. P-values $<0.05$ were considered significant.

\section{RESULTS}

We identified 109 women with T1DM referred to the Clinical Department for pregnancy planning or while pregnant. We excluded women who miscarried $(n=6)$, women who missed their post-delivery visit and those who's outcome data was missing $(n=22)$. In the analysis, we included 81 singleton pregnancies. We were unable to analyze miscarriages as an outcome because the data was incomplete.

There were 36 (44.4\%) women planning to become pregnant. Most of these were treated with CSII before conception and then during the pregnancy. Only six women were treated with an MDI regimen during the planning stage and three were then switched to CSIl after conception. Conversely, 31 (68.9\%) women who did not plan pregnancy were on an MDI regimen before conception. Most were then switched to CSII for the duration of the pregnancy. The insulin treatment and pregnancy planning are shown in detail in Table 1. During pregnancy, only 11 patients (13.6\%) remained on an MDI regimen, while 70 (86.4\%) were treated with CSII. There were 28 (40\%) patients on insulin pumps who used CGM sensors. A pump with automatic PLGS was available to 13 CGM users. Subsequent comparisons are between MDI, CSII without CGM and CSII with CGM groups. As an auxiliary analysis, we report comparisons between CSII patients using CGM with and without automatic insulin delivery suspension feature implemented in Medtronic $640 \mathrm{G}$ pump.

The clinical characteristics of the study participants are provided in Table 2. Briefly, the median age was 29 years and

Table 1. Methods of insulin treatment before and during pregnancy in the study group

\begin{tabular}{|l|c|c|c|c|}
\hline Treatment & \multicolumn{2}{|c|}{ Planning, $\mathbf{n = 3 6}(\mathbf{4 4 . 4 \% )}$} & \multicolumn{2}{c|}{ Not planning, $\mathbf{n = 4 5}(\mathbf{5 5 . 6 \% )}$} \\
\hline & Before pregnancy & During pregnancy & Before pregnancy & \multicolumn{1}{c|}{ During pregnancy } \\
\hline MDI, $n(\%)$ & $6(16.7)$ & $3(8.3)$ & $31(68.9 \%)$ & $8(17.8)$ \\
\hline CSII, $n(\%)$ & $30(83.3)$ & $33(91.7)$ & $14(31.1 \%)$ & $37(82.2)$ \\
\hline
\end{tabular}

$\mathrm{MDI}$ - multiple daily injections; CSII — continuous subcutaneous insulin infusion 


\begin{tabular}{|c|c|c|c|c|c|}
\hline Characteristic & $\begin{array}{c}\text { All } \\
(n=81)\end{array}$ & $\begin{array}{c}\text { CSII+CGM } \\
(n=28)\end{array}$ & $\begin{array}{l}\text { CSII-CGM } \\
(n=42)\end{array}$ & $\begin{array}{c}\text { MDI } \\
(n=11)\end{array}$ & p-value \\
\hline Age [years], median (IQR) & $29(27,33)$ & $30(27,33)$ & $28(26,33)$ & $29(27,32)$ & 0.314 \\
\hline Diabetes duration (years), median (IQR) & $15(9,18)$ & $13(9,19)$ & $16(10,20)$ & $12(3,17)$ & 0.117 \\
\hline BMI before conception $\left[\mathrm{kg} / \mathrm{m}^{2}\right]$, median (IQR) & $22.5(21.0,25.8)$ & $21.9(20.6,24.5)$ & $23.0(21.8,26.7)$ & $22.9(19.8,24.6)$ & 0.388 \\
\hline Systolic blood pressure [mm Hg], median (IQR) & $125(114,135)$ & $124(110,141)$ & $127(121,134)$ & $113(106,121)$ & 0.069 \\
\hline Diastolic blood pressure [mm Hg], median (IQR) & $74(70,80)$ & $73(66,81)$ & $75(70,80)$ & $72(70,77)$ & 0.770 \\
\hline Pre-conception $\mathrm{HbA}_{1 c}(\%)$, median (IQR) & $6.5(6.1,7.4)$ & $6.2(6.0,7.1)$ & $6.7(6.2,7.7)$ & $6.4(5.8,8.0)$ & 0.081 \\
\hline $\begin{array}{l}\text { White's class: B, n (\%) } \\
\text { C, } n(\%) \\
D, R, F, n(\%)\end{array}$ & $\begin{array}{l}26(32.1) \\
27(33.3) \\
28(34.6)\end{array}$ & $\begin{array}{l}11(39.3) \\
10(35.7) \\
7(25.0)\end{array}$ & $\begin{array}{l}11(26.2) \\
14(33.3) \\
17(40.5)\end{array}$ & $\begin{array}{l}4(36.4) \\
3(27.2) \\
4(36.4)\end{array}$ & 0.680 \\
\hline Retinopathy, n (\%) & $17(21.0)$ & $5(17.9)$ & $11(26.2)$ & $1(9.1)$ & 0.590 \\
\hline Albuminuria, n (\%) & $3(3.7)$ & $1(3.6)$ & $2(4.8)$ & 0 & 1.000 \\
\hline Daily insulin dose before pregnancy (U), median (IQR) & $45(34,54)$ & $45(37,52)$ & $44(34,51)$ & $41(34,63)$ & 0.677 \\
\hline Pregnancy planning, $n$ (\%) & $36(44.4)$ & $12(42.9)$ & $21(50.0)$ & $3(27.3)$ & 0.931 \\
\hline
\end{tabular}

$\mathrm{BMI}$ - body mass index; $\mathrm{HbA}_{1 \mathrm{c}}$ - glycated hemoglobin $\mathrm{A}_{1 \mathrm{c}} ; \mathrm{IQR}$ - interquartile range

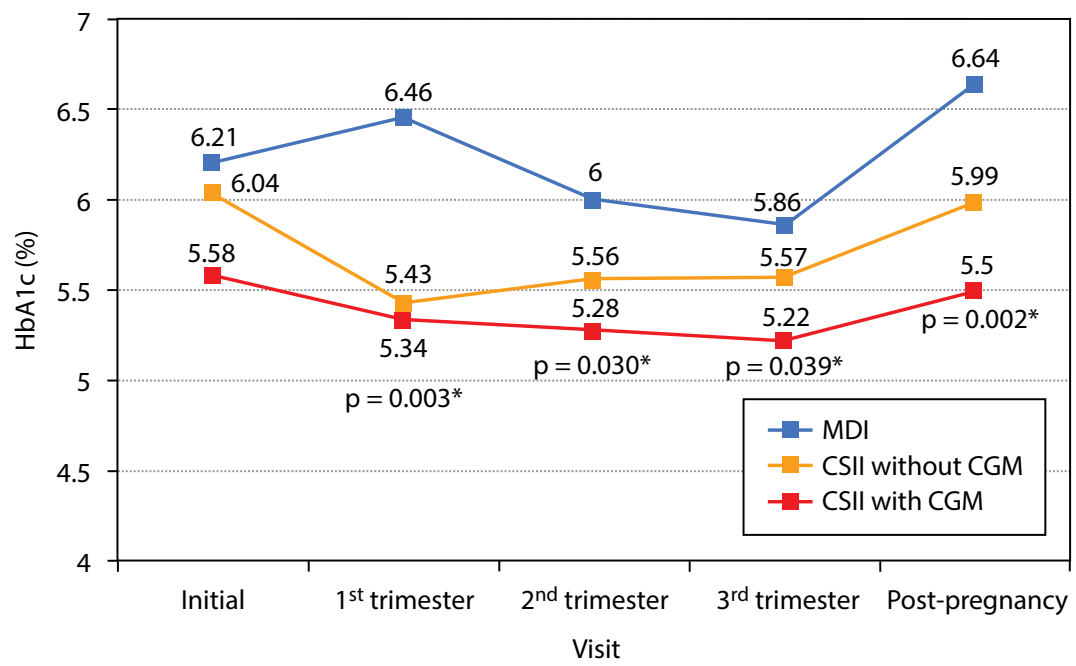

Figure 1. $\mathrm{HbA}_{1 \mathrm{c}}$ levels throughout pregnancy

T1DM duration was 15 years, without clinically significant differences between the treatment groups. The distribution of White's classes was not different ( $p$-value for differences between treatments was 0.68). The patients before conception had predominantly normal body weight. The patients were characterized by well-controlled diabetes, with median $\mathrm{HbA}_{1 \mathrm{c}}$ level before pregnancy (and before pregnancy planning) $6.5 \%$. Differences between median baseline $\mathrm{HbA}_{1 \mathrm{c}}$ among treatment groups were not statistically significant $(p=0.081)$.

Pregnancy planning was associated with $\mathrm{HbA} 1 \mathrm{c}$ lower by $0.9 \%$ at the initial visit $(p<0.001)$ and in the first trimester $(p<0.001)$. The difference became small $(0.1-0.3 \%)$ and not statistically significant in the second and third trimester or after delivery. To account for some of these imbalances in the frequency of pregnancy planning between treatment groups, especially in the MDI group, estimated mean levels of $\mathrm{HbA}_{1 \mathrm{c}}$ in subsequent analyses are adjusted for pregnancy planning. The $\mathrm{HbA}_{1 \mathrm{c}}$ levels throughout the pregnancy are shown and compared in Figure 1. On the first pregnancy visit, the women using sensors had already lower $\mathrm{HbA}_{1 \mathrm{c}^{\prime}}$ although the difference did not reach statistical significance (5.6\% in CGM users vs $6.0 \%$ and $6.2 \%$ in CSII and MDI groups, respectively, $\mathrm{p}=0.149)$. Women using CGM during pregnancy had the lowest $\mathrm{HbA}_{1 c}(5.3 \%, 5.3 \%$ and $5.2 \%$ in the $1^{\text {st }}, 2^{\text {nd }}$ and $3^{\text {rd }}$ trimester, respectively). After the pregnancy, the $\mathrm{HbA}_{1 \mathrm{c}}$ increased to $5.5 \%$ within 3-6 months. In the CSII group not using $\mathrm{CGM}, \mathrm{HbA}_{1 \mathrm{c}}$ was $5.4 \%, 5.6 \%$ and $5.6 \%$ in the first, second and third trimester, respectively, increasing to $6.0 \%$ after the pregnancy ended. The highest $\mathrm{HbA}_{1 \mathrm{c}}$ was 
observed in the MDI group: $6.5 \%, 6.0 \%$ and $5.9 \%\left(1^{\text {st }}, 2^{\text {nd }}\right.$, $3^{\text {rd }}$ trimester) and $6.6 \%$ during the postpartum visit. The differences between the study groups were statistically significant: $p=0.003, p=0.030, p=0.039$ and $p=0.002$ for the first, second and third trimesters, as well as post-pregnancy time points, respectively.

To further explore the impact of the use of CGM during pregnancy, we compared patients who did not use CGM against patients using CGM with and without the automatic insulin delivery suspension. Although patients who received pumps with PLGS had numerically (but not significantly) higher $\mathrm{HbA}_{1 \mathrm{c}}$ measured during the first visit (5.7\%) than women using CSIl without this function (5.3\%), they achieved a significantly lower $\mathrm{HbA}_{1 \mathrm{c}}(5.1 \%)$ than the other CSII patients using (5.3\%) or not using (5.6\%) CGM $(p=0.044)$ during the third trimester. The difference remained significant after pregnancy $(5.3 \%, 5.6 \%$ and $6.0 \%$, respectively, $\mathrm{p}=0.016$ ).

Pregnancy outcomes are summarized in Table 3. We did not find any significant differences in the pregnancy duration, gestational age at delivery, birth weight (also in an analysis limited to term pregnancies) or macrosomia risk. A composite outcome of macrosomia and preterm birth, as a preterm birth may be considered a proxy of macrosomia, did not reach statistical significance $(p=0.23)$, although there was numerically lower risk of this outcome among patients that used CGM (28.6\% versus $45.2 \%$ in pump users and $54.5 \%$ in the MDI group). The risk of macrosomia in the study cohort was high, $19.7 \%$, despite good glycemic control, especially in the third trimester. There were four (4.9\%) cases of birth weight $>4,500 \mathrm{~g}$. The risk of birth weight $<2,500 \mathrm{~g}$ was $7.4 \%$, without significant differences between study groups. There were 58 caesarian sections (71.6\%), with a significantly lower rate in the MDI group (36.4\% versus 69.1\% in CGM non-users and 89.3\% CGM users, respectively, $\mathrm{p}=0.004)$. We recorded eight congenital malforma- tions. Among them there were five cases of congenital heart abnormalities- one case of patent foramen ovale, one case of atrioventricular septum defect and aortic coarctation associated with trisomy 21 and one case of benign right ventricular hypertrophy with mild stenosis of the pulmonary valve, one case of ventricular septum defect and one case of Fallot's tetralogy. Other congenital malformations concerned two genetically determined syndromes - a case of Beckwith-Wiedemann syndrome (BWS) characterized by an enlarged tongue, umbilical hernia, hyperplasia, neonatal hypoglycemia and a case of multiple birth defects including atresia of the pulmonary trunk, major aortopulmonary collateral arteries (MAPCAS), ventricular septal defect, Fallot's tetralogy. We recorded one case of congenital limb defect. All pregnancies with defects were unplanned. There were no stillbirths or early hospital deaths in the study group. We did not observe significant differences in birth weight or a macrosomia risk between CGM users with and without automatic insulin delivery suspension.

\section{DISCUSSION}

In our present study, we evaluated the role of CGM technology in the management of T1DM in women during pregnancy. We observed that the use of CGM was associated with lower $\mathrm{HbA} 1 \mathrm{c}$ levels during pregnancy and this lasted for some time after delivery. In the third trimester, the difference in $\mathrm{HbA}_{1 \mathrm{c}}$ level reached $0.4 \%$ between CSII-treated women using and not using CGM. The CGM users compared to women treated with MDI had a $0.7 \%$ lower $\mathrm{HbA} 1 \mathrm{c}$ level. These results of using CGM during pregnancy in women with preexisting T1DM were comparable to some earlier published data [17]. It was described in an Irish cohort that CGM use was associated with improved glycemic control in the third trimester and lower birth weights and reduced risk of macrosomia. A separate observational study depicted a similar improvement of glycemic control parameters in

\begin{tabular}{|l|c|c|c|c|}
\hline \begin{tabular}{l} 
Table 3. Pregnancy outcomes in the study group \\
\hline Outcome
\end{tabular} & $\begin{array}{c}\text { CSII + CGM } \\
(\mathbf{n = 2 8 )}\end{array}$ & $\begin{array}{c}\text { CSII-CGM } \\
(\mathbf{n = 4 2 )}\end{array}$ & $\begin{array}{c}\text { MDI } \\
(\mathbf{n}=\mathbf{1 1})\end{array}$ & p-value \\
\hline Gestational age at delivery (years), median (IQR) & $38(38,39)$ & $38(36,39)$ & $38(35,39)$ & 0.156 \\
\hline Maternal weight gain [kg], median (IQR) & $17(13,21)$ & $16(14,18)$ & $14(11,17)$ & 0.239 \\
\hline Birth weight (g), median (IQR) & $3535(3245,3965)$ & $3580(3160,3800)$ & $3250(2700,4100)$ & 0.510 \\
\hline Birth weight in term pregnancies [g], median (IQR) & $3585(3300,4000)$ & $3730(3340,3940)$ & $3515(3195,4135)$ & 0.780 \\
\hline Preterm births, $\mathrm{n}(\%)$ & $2(7.1)$ & $12(28.6)$ & $3(27.2)$ & 0.084 \\
\hline Macrosomia $(>4,000 \mathrm{~g}), \mathrm{n}(\%)$ & $6(21.4)$ & $7(16.7)$ & $3(27.2)$ & 0.710 \\
\hline Low birth weight $(<2,500 \mathrm{~g}), \mathrm{n}(\%)$ & $1(3.6)$ & $3(7.1)$ & $2(18.2)$ & 0.276 \\
\hline Caesarian section, $\mathrm{n}(\%)$ & $25(89.3)$ & $29(69.1)$ & $4(36.4)$ & $0.004^{*}$ \\
\hline Birth defect, $\mathrm{n}(\%)$ & $3(10.7)$ & $3(7.1)$ & $1(9.1)$ & 0.870 \\
\hline
\end{tabular}


pregnant women with diabetes due to the use of CGM [18]. We also observed that pumps augmented with CGM with PLGS seemed to help women to achieve even better glycemic control as assessed by $\mathrm{HbA}_{1 \mathrm{c}}$.

In the third trimester, the difference in $\mathrm{HbA}_{1 \mathrm{c}}$ between PLGS pump users and the users of other pumps with CGM was $0.2 \%$ lower for PLGS users as compared to MDI and not CGM users. Similarly, to a recently published study, we have also shown a favorable reduction in $\mathrm{HbA}_{1 c}$ levels when using an insulin pump compared to MDI [19]. We were unable to demonstrate a reduction in glycemic variability because the CGM was not used in the whole observed group.

With this limited sample size, we were unable to detect significant differences in maternal or fetal complications of pregnancy. Despite a very good glycemic control, the risk of macrosomia (> 4,000 g) remained high reaching $19.7 \%$. No significant differences were seen in the risk of this complication between the study groups.

The current findings are concordant with our previous publications [3, 12]. We were able to confirm that pregnancy planning leads to better glycemic control throughout the pregnancy regardless of the insulin treatment regimen. We also noted a similar risk of macrosomia (19,7\%), as in our previous publication (22.7\%). It is unfortunate that despite technological improvements and strict standards of care, one in five pregnancies of women with T1DM results in the delivery of a macrosomic baby. Similar results were observed in an observational German study; they showed higher rates of large-for-gestational-age (LGA) neonates in the CSII-treated group vs MDI- treated group of pregnant women with T1DM, possibly mediated by excess maternal weight gain, which occurred more frequently in CSII users [20].

The recent results of CONCEPTT trial indicate that CGM use is associated with a statistically significant, but relatively small improvement in $\mathrm{HbA}_{1 \mathrm{c}}$ level. It has also been shown to improve the time in range spent on the glycemic target [9]. CGM use was associated with a reduced risk of macrosomia, shorter hospitalization, less neonatal intensive care admissions and a lower risk of neonatal hypoglycemia. The widespread use of CGM during pregnancy may induce cost savings in the healthcare system due to the reduced risk of hyperglycemic-related complications. Women enrolled in the trial were characterized by a higher preconception $\mathrm{HbA}_{1 c^{\prime}}$ as well as $\mathrm{HbA}_{1 \mathrm{c}}$ during pregnancy. Similar levels of $\mathrm{HbA}_{1 \mathrm{c}}$ were recorded in an observational study in Sweden [21], which compared a real-time and intermittent CGM during pregnancy. In our study, $\mathrm{HbA}_{1 \mathrm{c}}$ declined to levels typically observed in non-diabetic individuals during the second and third trimesters. This may explain the lower risk of macrosomia observed in our study and a small number of cases of birth weight exceeding $4.5 \mathrm{~kg}$.
In this study, we attempted to compare the use of pumps augmented with CGM versus the use of pumps without CGM and MDI regimen. The observational study design does not allow for any firm conclusions, however, there was a clear trend towards better glycemic control among the users of insulin pumps with CGM.

There were several limitations in our study that need to be acknowledged. First, the time frame of the study and its sample size is limited. Subsequently, we were not able to perform a regression analysis for important pregnancy outcomes, such as birth weight. The study lacked power to detect differences in infrequent complications such as congenital malformations or deaths. Treatment allocation was not randomized, and in the case of an MDI regimen, it was specifically provided to women not accepting CSII for various reasons. Such a study may be considered a hypothesis-generating one rather than providing definitive conclusions. Nevertheless, reporting real-world data, such as this and related to the important problems of T1DM complicated pregnancy, supplements the results of randomized clinical trials. Another limitation of the study was the inability to compare modern glycemic control parameters.

\section{CONCLUSIONS}

In summary, the use of pumps integrated with CGM may improve glycemic control as estimated with $\mathrm{HbA}_{1 \mathrm{c}}$. Despite the use of a new generation of treatment, the proportion of macrosomia remains high in T1DM complicated pregnancies.

\section{Acknowledgements}

We would like to thank The Great Orchestra of Christmas Charity.

\section{Conflict of interest}

The authors have nothing to declare.

\section{Ethical Approval}

Informed consent was obtained from each participant. The study was approved by the institutional ethics committee. All procedures performed in this study were in accordance with the ethical standards of the institutional research committee and with the 1964 Helsinki declaration and its later amendments or comparable ethical standards.

\section{Contribution statement}

KC, JS, MTM: study design, protocol development; IL, KC: searching medical databases; KC, BM, JS: data analysis; IL, KC, PW, JS, MTM: research data, data interpretation; IL, KC, JS, MTM: writing the manuscript; MTM: critical review of the manuscript; KC, MTM: project coordination, final approval of the manuscript. JS is the guarantor of the data and, as 
such, had full access to all the data in the study and takes responsibility for the integrity of the data and the accuracy of the data analysis.

\section{REFERENCES}

1. Owens LA, Avalos G, Kirwan B, et al. ATLANTIC DIP: closing the loop: a change in clinical practice can improve outcomes for women with pregestational diabetes. Diabetes Care. 2012; 35(8): 1669-1671, doi: 10.2337/dc12-0120, indexed in Pubmed: 22826448.

2. Tennant PWG, Glinianaia SV, Bilous RW, et al. Pre-existing diabetes, maternal glycated haemoglobin, and the risks of fetal and infant death: a population-based study. Diabetologia. 2014; 57(2): 285-294, doi: 10.1007/s00125-013-3108-5, indexed in Pubmed: 24292565.

3. Cyganek K, Skupien J, Katra B, et al. Risk of macrosomia remains glucose-dependent in a cohort of women with pregestational type 1 diabetes and good glycemic control. Endocrine. 2017; 55(2): 447-455, doi: 10.1007/s12020-016-1134-z, indexed in Pubmed: 27726091.

4. Kelstrup L, Clausen TD, Mathiesen ER, et al. High prevalence of type 2 diabetes and pre-diabetes in adult offspring of women with gestational diabetes mellitus or type 1 diabetes: the role of intrauterine hyperglycemia. Diabetes Care. 2008; 31(2): 340-346, doi: 10.2337/dc07-1596, indexed in Pubmed: 18000174.

5. Hillier TA, Pedula KL, Schmidt MM, et al. Childhood obesity and metabolic imprinting: the ongoing effects of maternal hyperglycemia. Diabetes Care. 2007; 30(9): 2287-2292, doi: 10.2337/dc06-2361, indexed in Pubmed: 17519427.

6. Rijpert M, Evers IM, de Vroede MA, et al. Risk factors for childhood overweight in offspring of type 1 diabetic women with adequate glycemic control during pregnancy: Nationwide follow-up study in the Netherlands. Diabetes Care. 2009; 32(11): 2099-2104, doi: 10.2337/dc09-0652, indexed in Pubmed: 19651922.

7. Murphy HR, Bell R, Cartwright C, et al. Improved pregnancy outcomes in women with type 1 and type 2 diabetes but substantial clinic-to-clinic variations: a prospective nationwide study. Diabetologia. 2017;60(9): 16681677, doi: 10.1007/s00125-017-4314-3, indexed in Pubmed: 28597075.

8. Jendle J, Smith-Palmer J, Delbaere A, et al. Cost-Effectiveness Analysis of Sensor-Augmented Insulin Pump Therapy with Automated Insulin Suspension Versus Standard Insulin Pump Therapy in Patients with Type 1 Diabetes in Sweden. Diabetes Ther. 2017; 8(5): 1015-1030, doi: 10.1007/s13300-017-0294-z, indexed in Pubmed: 28871565.

9. Feig DS, Donovan LE, Corcoy R, et al. CONCEPTT Collaborative Group. Continuous glucose monitoring in pregnant women with type 1 diabetes (CONCEPTT): a multicentre international randomised controlled trial. Lancet. 2017; 390(10110): 2347-2359, doi: 10.1016/S01406736(17)32400-5, indexed in Pubmed: 28923465.
10. Broughton C, Douek I. An overview of the management of diabetes from pre-conception, during pregnancy and in the postnatal period. Clinical Medicine. 2019; 19(5):399-402, doi: 10.7861/clinmed.2018-0288.

11. American Diabetes Association. Management of Diabetes in Pregnancy: Standards of Medical Care in Diabetes-2020. American Diabetes Association. Diabetes Care. 2020; 43(Suppl 1): S183-S192, doi: 10.2337/dc20-S014, indexed in Pubmed: 31862757.

12. Cyganek K, Hebda-Szydlo A, Katra B, et al. Glycemic control and selected pregnancy outcomes in type 1 diabetes women on continuous subcutaneous insulin infusion and multiple daily injections: the significance of pregnancy planning. Diabetes Technol Ther. 2010; 12(1): 41-47, doi: 10.1089/dia.2009.0081, indexed in Pubmed: 20082584.

13. 2016 Guidelines on the management of diabetic patients. A position of Diabetes Poland. Clinical Diabetol. 2016; 5(Suppl A): A46-A48.

14. 2020 Guidelines on the management of diabetic patients. A position of Diabetes Poland. Clin Diabetol 2020; 9, 1:64-68. doi: 10.5603/DK.2020.0001. 2020; 9(1): 64-68, doi: 10.5603/DK.2020.0001.

15. Wender-Ożegowska E, Bomba-Opoń D, Brazzert J, et al. Standards of Polish Society of Gynecologists and Obstetricians in management of women with diabetes. Ginekol Pol. 2018; 89(6): 341-350, doi: 10.5603/GP.a2018.0059, indexed in Pubmed: 30010185.

16. Advani A. Positioning time in range in diabetes management. Diabetologia. 2020; 63(2): 242-252, doi: 10.1007/s00125-019-05027-0, indexed in Pubmed: 31701199.

17. Murphy HR, Rayman G, Lewis K, et al. Effectiveness of continuous glucose monitoring in pregnant women with diabetes: randomised clinical trial. BMJ. 2008; 337: a1680, doi: 10.1136/bmj.a1680, indexed in Pubmed: 18818254

18. Polsky S, Garcetti R, Pyle L, et al. Continuous glucose monitor use with and without remote monitoring in pregnant women with type 1 diabetes: A pilot study. PLoS One. 2020; 15(4): e0230476, doi: 10.1371/journal. pone.0230476, indexed in Pubmed: 32298269.

19. Jotic A, Milicic T, Lalic K, et al. Evaluation of Glycaemic Control, Glucose Variability and Hypoglycaemia on Long-Term Continuous Subcutaneous Infusion vs. Multiple Daily Injections: Observational Study in Pregnancies With Pre-Existing Type 1 Diabetes. Diabetes Ther. 2020; 11(4): 845-858, doi: 10.1007/s13300-020-00780-7, indexed in Pubmed: 32060738.

20. Hauffe F, Schaefer-Graf UM, Fauzan R, et al. Higher rates of large-for-gestational-age newborns mediated by excess maternal weight gain in pregnancies with Type 1 diabetes and use of continuous subcutaneous insulin infusion vs multiple dose insulin injection. Diabet Med. 2019; 36(2): 158-166, doi: 10.1111/dme.13861, indexed in Pubmed: 30698863.

21. Kristensen K, Ögge LE, Sengpiel V, et al. Continuous glucose monitoring in pregnant women with type 1 diabetes: an observational cohort study of 186 pregnancies. Diabetologia. 2019; 62(7): 1143-1153, doi: 10.1007/s00125-019-4850-0, indexed in Pubmed: 30904938. 